\title{
Estimates of Some Operators on One-Sided Weighted Morrey Spaces
}

\author{
Shaoguang Shi and Zunwei Fu \\ Department of Mathematics, Linyi University, Linyi 276005, China \\ Correspondence should be addressed to Zunwei Fu; zwfu@mail.bnu.edu.cn \\ Received 13 August 2013; Revised 30 September 2013; Accepted 3 October 2013 \\ Academic Editor: Bashir Ahmad
}

Copyright (C) 2013 S. Shi and Z. Fu. This is an open access article distributed under the Creative Commons Attribution License, which permits unrestricted use, distribution, and reproduction in any medium, provided the original work is properly cited.

A version of one-sided weighted Morrey space is introduced. The boundedness of some classical one-sided operators in harmonic analysis and PDE on these spaces are discussed, including the Riemann-Liouville fractional integral.

\section{Introduction}

The reasons to study one-sided operators involve not only the generalization of the theory of two-sided operators but also the requirements of ergodic theory [1]. The well-known Riemann-Liouville fractional integral can be viewed as the one-sided version of Riesz potential (the solution of Laplace equation) in harmonic analysis and PDE $[2,3]$. The study of weighted theory for one-sided operators was first introduced by Sawyer [4] and many authors thereafter [5-10]. Many of their results show that, for a class of smaller operators (one-sided operators) and a class of wider weights (one-sided weights), many of the famous findings of harmonic analysis still hold.

The study of one-sided spaces emerged naturally alongside the study of one-sided operators. In one previous study, the authors studied one-sided BMO spaces associated with one-sided sharp functions and their relationship to good weights for the one-sided Hardy-Littlewood maximal functions [9]. Aimar and Crescimbeni [11] further investigated the structures of these one-sided regular functions and their basic properties. Other classical works regarding one-sided spaces have also been published [12-14].

A version of one-sided weighted Morrey spaces and Campanato spaces is introduced in this paper. The boundedness of some one-sided operators and its effects on these spaces are investigated. First recall some definitions of the classical Campanato spaces and Morrey spaces.
Let $-1 / p \leq \beta<1$ and $1 \leq p<\infty$. Then the Campanato space $\mathscr{C}^{p, \beta}(\mathbb{R})$ can be defined using the following norm

$$
\begin{aligned}
\|f\|_{\mathscr{C} P, \beta(\mathbb{R})} & =\sup _{\mathscr{I}}\|f\|_{\mathscr{G}, \beta(\mathscr{F})} \\
& =\sup _{\mathscr{I}} \frac{1}{|\mathcal{F}|^{\beta}}\left(\frac{1}{|\mathscr{I}|} \int_{\mathscr{I}}\left|f-f_{\mathscr{I}}\right|^{p} d x\right)^{1 / p},
\end{aligned}
$$

where $f_{\mathscr{I}}=(1 /|\mathscr{I}|) \int_{\mathscr{I}} f(x) d x, \mathscr{I}$ denotes an interval contained in $\mathbb{R}$, and $|\mathscr{F}|$ is the Lebesgue measure of $\mathscr{I}$. The excellent structures of Campanato spaces render them useful in the study of the regularity theory of PDEs. They allow the user to determine an integral characterization of the spaces of Hölder continuous functions. This allows generalization of the classical Sobolev embedding theorems; see [15-17], for example. It is also well known that $\mathscr{C}^{1,1 / p-1}(\mathbb{R})$ is the dual space of Hardy space $H^{p}(\mathbb{R})$ when $0<p<1$ [18]. There has been also a recent account of the theory on Campanato spaces [19-21]. The original form of classical Morrey space was first introduced by Morrey Jr. [22] to investigate the local behavior of solutions to the second order elliptic PDEs,

$$
\begin{aligned}
\|f\|_{\mathscr{U}^{p, \beta}(\mathbb{R})} & =\sup _{\mathscr{I}}\|f\|_{\mathscr{M}^{p, \beta}(\mathcal{F})} \\
& =\sup _{\mathscr{I}} \frac{1}{|\mathscr{I}|^{\beta}}\left(\frac{1}{|\mathscr{I}|} \int_{\mathscr{I}}|f(x)|^{p} d x\right)^{1 / p} .
\end{aligned}
$$


It is obvious that $\mathscr{M}^{p,-1 / p}(\mathbb{R})=L^{p}(\mathbb{R})$. Many properties of solutions to PDEs are concerned with the boundedness of some operators on Morrey type spaces. In fact, the better inclusion between the Morrey and the Hölder spaces permits obtaining higher regularity of the solutions to different elliptic and parabolic boundary problems. In recent years, there has been an explosion of interest in the study of the boundedness of operators on Morrey type spaces [23-25].

The study of weighted estimates and their effects on these spaces is important to harmonic analysis. Weighted inequalities arise naturally in Fourier analysis, but their use is best justified by the variety of applications in which they appear. For example, the theory of weights plays an important role in the study of boundary value problems inherent in Laplace's equations on Lipschitz domains. Many authors are interested in the study of the events that occur when the weight function belongs to one of the Muckenhoupt classes. Let $1<p<\infty$. The Muckenhoupt class $A_{p}$ [26] consists of all positive locally integrable functions $w$ for which

$$
\sup _{\mathscr{I}}\left(\frac{1}{|\mathscr{I}|} \int_{\mathscr{I}} w(x) d x\right)\left(\frac{1}{|\mathscr{I}|} \int_{\mathscr{I}} w(x)^{1-p^{\prime}} d x\right)^{p-1}<\infty,
$$

where $1 / p+1 / p^{\prime}=1$. We say that $w \in A_{1}$ if there is a constant $C>0$ such that $M w(x) \leq C w(x)$. Here $M$ is the HardyLittlewood maximal operator

$$
M f(x)=\sup _{h>0} \frac{1}{h} \int_{x-h}^{x+h}|f(y)| d y .
$$

The study of weights for one-sided operators is motivated by their natural emergence in harmonic analysis. For example, certain measures are required when the one-sided HardyLittlewood maximal operators [4]

$$
\begin{aligned}
& M^{+} f(x)=\sup _{h>0} \frac{1}{h} \int_{x}^{x+h}|f(y)| d y, \\
& M^{-} f(x)=\sup _{h>0} \frac{1}{h} \int_{x-h}^{x}|f(y)| d y
\end{aligned}
$$

arising in the ergodic maximal function are treated. The classical Dunford-Schwartz ergodic theorem can be considered the first result regarding weights for these operators. In [4], Sawyer introduced the one-sided $A_{p}$ classes $A_{p}^{+}$and $A_{p}^{-}$; they are defined by the following conditions:

$$
\begin{aligned}
A_{p}^{+}: A_{p}^{+}(w):= & \sup _{a<b<c} \frac{1}{(c-a)^{p}} \\
& \times \int_{a}^{b} w(x) d x\left(\int_{b}^{c} w(x)^{1-p^{\prime}} d x\right)^{p-1} \\
< & \infty, \\
A_{p}^{-}: A_{p}^{-}(w):= & \sup _{a<b<c} \frac{1}{(c-a)^{p}} \\
& \times \int_{b}^{c} w(x) d x\left(\int_{a}^{b} w(x)^{1-p^{\prime}} d x\right)^{p-1} \\
< & \infty,
\end{aligned}
$$

when $1<p<\infty$; also, for $p=1$,

$$
A_{1}^{+}: M^{-} w \leq C w, \quad A_{1}^{-}: M^{+} w \leq C w
$$

for some constant $C$. The smallest constant $C$ was denoted by $A_{1}^{+}(w)\left(A_{1}^{-}(w)\right) . A_{p}^{+}(w)\left(A_{p}^{-}(w)\right), p \geq 1$, will be called the $A_{p}^{+}\left(A_{p}^{-}\right)$constant of $w$.

Theorem 1 (see [4]). Let $1<p<\infty$. Then there exists $C>0$ such that the inequality

$$
\left\|M^{+} f\right\|_{L^{p}(w)} \leq C\|f\|_{L^{p}(w)}
$$

holds for all $f \in L^{p}(w)$ if and only if $w \in A_{p}^{+}$.

Remark 2. Similar results can be obtained for the left-handside operator by changing the condition $A_{p}^{+}$to $A_{p}^{-}$.

A function $K$ is called a one-sided Calderón-Zygmund kernel (OCZK) if $K$ satisfies

$$
\begin{gathered}
\left|\int_{a<|x|<b} K(x) d x\right| \leq C, \quad 0<a<b, \\
|K(x)| \leq \frac{C}{|x|}, \quad x \neq 0, \\
|K(x-y)-K(x)| \leq \frac{C|y|}{|x|^{2}}, \quad|x|>2|y|>0
\end{gathered}
$$

with support in $\mathbb{R}^{-}=(-\infty, 0)$ or $\mathbb{R}^{+}=(0,+\infty)$. Equation (10) is also called the size condition for $K$ and (11) is the continuous condition for $K$. An example of such a kernel is

$$
K(x)=\frac{\sin (\log |x|)}{(x \log |x|)} \chi_{(-\infty, 0)}(x),
$$

where $\chi_{E}$ denotes the characteristic function of a set $E$. Aimar et al. [5] studied the one-sided Calderón-Zygmund singular integrals which were defined by

$$
\begin{aligned}
& T^{+} f(x)=\lim _{\varepsilon \rightarrow 0^{+}} \int_{x+\varepsilon}^{\infty} K(x-y) f(y) d y \\
& T^{-} f(x)=\lim _{\varepsilon \rightarrow 0^{+}} \int_{-\infty}^{x-\varepsilon} K(x-y) f(y) d y,
\end{aligned}
$$

where the kernels $K$ are OCZKs.

The one-sided $A_{p}$ classes not only control the boundedness of one-sided Hardy-Littlewood maximal operators, but also serve as the right weight classes for one-sided singular integral operators. They also appear in PDEs [27].

Theorem 3 (see [5]). Let $1<p<\infty$, and let $K$ be an OCZK with support in $\mathbb{R}^{-}=(-\infty, 0)$. Then $T^{+}$is bounded on $L^{p}(w)$ if $w \in A_{p}^{+}$.

Also, a result concerning the converse of Theorem 3 is given in [5].

In addition to singular integral operators, fractional integral operators also play an important role in harmonic analysis. The problem of fractional derivation was an early impetus 
to study fractional integrals [6]. In addition to their contributions to harmonic analysis, fractional integrals also play an essential role in many fields. The Hardy-Littlewood-Sobolev inequality of fractional integral is still an indispensable tool in the establishment of time-space estimates for the heat semigroup of nonlinear evolution equations. Let $0<\alpha<1$; the one-sided fractional maximal operator and the one-sided fractional integrals were defined by

$$
\begin{gathered}
M_{\alpha}^{+} f(x)=\sup _{h>0} \frac{1}{h^{1-\alpha}} \int_{x}^{x+h}|f(y)| d y, \\
M_{\alpha}^{-} f(x)=\sup _{h>0} \frac{1}{h^{1-\alpha}} \int_{x-h}^{x}|f(y)| d y, \\
I_{\alpha}^{+} f(x)=\int_{x}^{\infty} \frac{f(y)}{(y-x)^{1-\alpha}} d y, \\
I_{\alpha}^{-} f(x)=\int_{-\infty}^{x} \frac{f(y)}{(y-x)^{1-\alpha}} d y,
\end{gathered}
$$

respectively. $I_{\alpha}^{+}$and $I_{\alpha}^{-}$are also called the Rieman-Liouville and the Weyl fractional integral operators. The boundedness of $M_{\alpha}^{+}$was determined by Andersen and Sawyer [6].

Theorem 4 (see [6]). Let $1<p<q<\infty, 1 / p-1 / q=\alpha$, and $w \in A_{(p, q)}^{+}$. Then there exists $C>0$ such that

$$
\begin{aligned}
& \text { (a) }\left\|M_{\alpha}^{+} f w\right\|_{L^{q}} \leq C\|f w\|_{L^{p}} ; \\
& \text { (b) }\left\|I_{\alpha}^{+} f w\right\|_{L^{q}} \leq C\|f w\|_{L^{p}} .
\end{aligned}
$$

The weight conditions $A_{(p, q)}^{+}$and $A_{(p, q)}^{-}$are denoted by

$$
\begin{aligned}
& A_{(p, q)}^{+}: \frac{1}{(c-a)^{1-\alpha}}\left(\int_{a}^{b} w^{q}\right)^{1 / q}\left(\int_{b}^{c} w^{-p^{\prime}}\right)^{1 / p^{\prime}} \leq C, \\
& A_{(p, q)}^{-}: \frac{1}{(c-a)^{1-\alpha}}\left(\int_{b}^{c} w^{q}\right)^{1 / q}\left(\int_{a}^{b} w^{-p^{\prime}}\right)^{1 / p^{\prime}} \leq C
\end{aligned}
$$

for all $a<b<c \in \mathbb{R}, 1<p<q$ and $1 / p-1 / q=\alpha$.

The one-sided Campanato space and one-sided Morrey space can now be introduced.

Definition 5. Let $-1 / p \leq \beta<1$ and $1 \leq p<\infty$. A locally integrable function $f$ is said to belong to the one-sided weighted Campanato space $\mathscr{C}_{p, \beta}^{+}(w)$ if

$$
\begin{aligned}
\|f\|_{\mathscr{C}_{p, \beta}^{+}(w)}=\sup _{x_{0}} \sup _{h>0} \frac{1}{h^{\beta}} \\
\quad \times\left(\frac{1}{w\left(x_{0}-h, x_{0}\right)}\right. \\
\left.\quad \times \int_{x_{0}}^{x_{0}+h}\left|f(y)-f_{\left(x_{0}, x_{0}+h\right)}\right|^{p} d y\right)^{1 / p}
\end{aligned}
$$$$
<\infty
$$

where $w\left(x_{0}-h, x_{0}\right)=\int_{x_{0}-h}^{x_{0}} w(x) d x, x_{0}$ is a real number.
When $\beta=0$ and $p=1, \mathscr{C}_{1,0}^{+}(w)$ coincides with the dual space of the one-sided weighted Hardy space $H_{+}^{1}(w)$ [28], which consists of certain classes of one-sided weighted BMO functions, see also $[11,13]$.

When $0<\beta<1$, and $p=1, \mathscr{C}_{1, \beta}^{+}(w)$ consists of all functions satisfying a weighted Lipschitz condition [13].

Case $\beta<0$ is addressed in the present work.

Definition 6. Let $-1 / p \leq \beta<0$ and $1 \leq p<\infty$. The onesided weighted Morrey space is defined by the norm

$$
\begin{aligned}
\|f\|_{\mathscr{M}_{p, \beta}^{+}(w)} & =\sup _{x_{0}} \sup _{h>0} \frac{1}{h^{\beta}}\left(\frac{1}{w\left(x_{0}-h, x_{0}\right)} \int_{x_{0}}^{x_{0}+h}|f(y)|^{p} d y\right)^{1 / p} \\
& <\infty .
\end{aligned}
$$

A standard calculation shows that $\mathscr{M}_{p, \beta}^{+}(w) \subseteq \mathscr{C}_{p, \beta}^{+}(w)$ in the sense that the following is true:

$$
\begin{array}{r}
\|f\|_{\mathscr{C}_{p, \beta}^{+}(w)} \\
\leq C \sup _{h>0} \frac{1}{h^{\beta}}\left(\frac{1}{w\left(x_{0}-h, x_{0}\right)} \int_{x_{0}}^{x_{0}+h}|f(y)-a|^{p} d y\right)^{1 / p}, \\
\forall a \in \mathbb{R}, \beta<0 .
\end{array}
$$

Section 2 outlines proof of the boundedness of some onesided operators mentioned above on $\mathscr{M}_{p, \beta}^{+}(w)$. In Section 3, the results in Section 1 are extended to a one-sided sublinear operator under specific size conditions, which were satisfied by many one-sided operators, including $M^{+}, T^{+}, M_{\alpha}^{+}$, and $I_{\alpha}^{+}$.

Throughout this paper, for $x_{0} \in \mathbb{R}$ and $h, \lambda>0$, unless otherwise stated, we will always denote that $I=\left(x_{0}, x_{0}+h\right)$, $I^{+}=\left(x_{0}+h, x_{0}+2 h\right), I^{-}=\left(x_{0}-h, x_{0}\right)$, and $\lambda I=\left(x_{0}, x_{0}+\lambda h\right)$. $C$ is a constant which may change from line to line.

\section{Main Results}

In this section, the boundedness of the one-sided operators mentioned in Section 1 and its effects on one-sided Morrey spaces are described. The primary results are formulated as follows.

Theorem 7. Let $-1 / p \leq \beta<0$ and $w \in A_{p}^{+}$. Then

(a) $M^{+}$is a bounded operator from $\mathscr{M}_{p, \beta}^{+}(w)$ to $\mathscr{C}_{p, \beta}^{+}(w)$ for $1<p<1 /(1+\beta)$;

(b) $T^{+}$is a bounded operator from $\mathscr{M}_{p, \beta}^{+}(w)$ to $\mathscr{C}_{p, \beta}^{+}(w)$ for $1<p<\infty$.

Theorem 7(a) is also true when $\mathscr{C}_{p, \beta}^{+}(w)$ is replaced by $\mathscr{M}_{p, \beta}^{+}(w)$ (see proof of Theorem 7). A corresponding substitution for Theorem 7(b) under certain assumption with respect to $p$ is given in Section 3 .

For the fractional case, the following is true. 
Theorem 8. Let $0<\alpha<1,1 / q=1 / p-\alpha,-1 / p \leq \beta<0$, and $w \in A_{(p, q)}^{+}$. Then

(a) $M_{\alpha}^{+}$is a bounded operator from $\mathscr{M}_{p, \beta}^{+}\left(w^{p}\right)$ to $\mathscr{C}_{q, \beta}^{+}\left(w^{q}\right)$ for $1<p<q<1 /(1+\beta)$;

(b) $I_{\alpha}^{+}$is a bounded operator from $\mathscr{M}_{p, \beta}^{+}\left(w^{p}\right)$ to $\mathscr{C}_{q, \beta}^{+}\left(w^{q}\right)$ for $1<p<1 / \alpha$.

First, some basic propositions of one-sided weight classes are selected for use in the analysis.

Lemma 9 (see [4]). (a)If $w \in A_{p}^{+}$, then $w \in A_{p-\varepsilon}^{+}$for some $\varepsilon>0$.

(b) $w \in A_{p}^{+}$for $1<p<\infty$ if and only if there exists $w_{1} \in$ $A_{1}^{+}$and $w_{2} \in A_{1}^{-}$such that $w=w_{1}\left(w_{2}\right)^{1-p}$.

(c)If $1 \leq p<\infty$, then $A_{p}=A_{p}^{+} \cap A_{p}^{-}, A_{p} \subset A_{p}^{+}$, and $A_{p} \subset A_{p}^{-}$.

(d) $A_{p}^{+} \subset A_{r}^{+}, A_{p}^{-} \subset A_{r}^{-}$if $1 \leq p \leq r$.

According to the definitions of $A_{p}^{+}$and $A_{(p, q)}^{+}$, the following relationship between these two classes can be assessed easily.

Proposition 10. Suppose $0<\alpha<1,1<p<q<\infty$, and $1 / p-1 / q=\alpha$; then the following statements are equivalent.
(a) $w \in A_{(p, q)}^{+}$.
(b) $w^{q} \in A_{q(1-\alpha)}^{+}$.
(c) $w^{q} \in A_{q}^{+}, w^{p} \in A_{p}^{+}$.
(d) $w^{-p^{\prime}} \in A_{1+\left(q / p^{\prime}\right)}^{+}$.

Proof. Proof is given only for $(a) \Leftrightarrow(b)$. Other cases are straightforward and can be described using Lemma 9 and the definitions of $A_{p}^{+}$and $A_{(p, q)}^{+}$.

(a) $\Rightarrow$ (b). If $w \in A_{(p, q)}^{+}$, we have

$$
\frac{1}{(c-a)^{1-\alpha}}\left(\int_{a}^{b} w^{q}\right)^{1 / q}\left(\int_{b}^{c} w^{-p^{\prime}}\right)^{1 / p^{\prime}} \leq C,
$$

which if combined $1 / p-1 / q=\alpha$ implies

$$
\begin{aligned}
& \frac{1}{(c-a)^{q(1-\alpha)}} \int_{a}^{b} w^{q}\left(\int_{b}^{c} w^{q\left(1-(q(1-\alpha))^{\prime}\right)}\right)^{q(1-\alpha)-1} \\
& =\frac{1}{(c-a)^{q(1-\alpha)}} \\
& \quad \times \int_{a}^{b} w^{q}\left(\int_{b}^{c} w^{q\left(\left(1-q^{\prime}\right) /\left(1-q^{\prime} \alpha\right)\right)}\right)^{q(1-\alpha)-1} \\
& =\frac{1}{(c-a)^{q(1-\alpha)}} \int_{a}^{b} w^{q}\left(\int_{b}^{c} w^{-p^{\prime}}\right)^{(p-1) /(1-p \alpha)} \\
& <\infty .
\end{aligned}
$$

Therefore $w^{q} \in A_{q(1-\alpha)}^{+}$. (b) $\Rightarrow$ (a). It is obvious by the reverse argument of (a) $\Rightarrow$ (b).

If $w(x) \in A_{p}$; then it is a doubling weight, that is, there exists $C>0$ such that

$$
\int_{a-2 h}^{a+2 h} w \leq C \int_{a-h}^{a+h} w
$$

for all $a \in \mathbb{R}$ and $h>0$. However, one-sided $A_{p}$ weights do not satisfy this property. But the weights $A_{p}^{+}$satisfy a onesided doubling condition.

Lemma 11 (see [29]). Let $w(x) \in A_{p}^{+}(p \geq 1)$. Then there exists a constant $C>0$ such that

$$
\int_{a-h}^{a+h} w \leq C \int_{a}^{a+h} w
$$

for all $a \in \mathbb{R}$ and $h>0$.

Like the one-sided doubling condition, the following proposition also plays an important role in the present arguments.

Proposition 12. Let $\lambda>0$ and $p, q \geq 1$. Then

(a) if $w \in A_{p}^{+}$, we have

$$
w\left((\lambda I)^{-}\right) \leq C \lambda^{p} w(I) ;
$$

(b) if $w \in A_{(p, q)}^{+}$, we have

$$
w\left((\lambda I)^{-}\right) \leq C \lambda w(I) .
$$

Proof. For the proof of (a), we first claim that

$$
\left(f_{I}\right)^{p} \leq C A_{p}^{+}(w)\left(\frac{1}{w\left(I^{-}\right)} \int_{I}|f(x)|^{p} w(x) d x\right) .
$$

In fact, we can apply Hölder's inequality with exponents $p$ and $p^{\prime}$ to get

$$
\begin{aligned}
\left(\frac{1}{|I|} \int_{I}|f(x)| d x\right)^{p} \\
=\left(\frac{1}{|I|} \int_{I}|f(x)| w(x)^{1 / p} w(x)^{-1 / p} d x\right)^{p} \\
\leq \frac{1}{|I|^{p}}\left(\int_{I}|f(x)|^{p} w(x) d x\right) \\
\quad \times\left(\int_{I} w(x)^{-p^{\prime} / p} d x\right)^{p / p^{\prime}} \\
=\left(\frac{1}{w\left(I^{-}\right)} \int_{I}|f(x)|^{p} w(x) d x\right) \\
\quad \times\left(\frac{1}{|I|} \int_{I^{-}} w(x) d x\right) \\
\quad \times\left(\frac{1}{|I|} \int_{I} w(x)^{-p^{\prime} / p} d x\right)^{p-1} \\
\leq C A_{p}^{+}(w)\left(\frac{1}{w\left(I^{-}\right)} \int_{I}|f(x)|^{p} w(x) d x\right) .
\end{aligned}
$$


Applying (26) to the function $f=\chi_{I}$ and putting $\lambda I$ in the place of $I$ in (26), we obtain

$$
w\left((\lambda I)^{-}\right) \leq A_{p}^{+}(w) \lambda^{p} w(I) \leq C \lambda^{p} w(I) .
$$

The proof of (b) is a byproduct of (a) and the fact that $w^{p} \in A_{p}^{+}$if $w \in A_{(p, q)}^{+}$.

Proof of Theorem 7. The proof of (a) is given first. Because $\mathscr{M}_{p, \beta}^{+}(w) \subseteq \mathscr{C}_{p, \beta}^{+}(w)$ when $\beta<0$, it is sufficient to prove that there exists $C>0$ such that

$$
\frac{1}{h^{\beta}}\left(\frac{1}{w\left(x_{0}-h, x_{0}\right)} \int_{x_{0}}^{x_{0}+h}\left|M^{+} f(x)\right|^{p} d x\right)^{1 / p} \leq C\|f\|_{\mathscr{U}_{p, \beta}^{+}(w)} .
$$

Decompose $f=f_{1}+f_{2}=f \chi_{2 I}+f \chi_{(2 I)^{c}}$ to obtain

$$
\begin{aligned}
& \frac{1}{h^{\beta}}(\left.\frac{1}{w\left(x_{0}-h, x_{0}\right)} \int_{x_{0}}^{x_{0}+h}\left|M^{+} f(x)\right|^{p} d x\right)^{1 / p} \\
& \leq \frac{1}{h^{\beta}}\left(\frac{1}{w\left(x_{0}-h, x_{0}\right)} \int_{x_{0}}^{x_{0}+h}\left|M^{+} f_{1}(x)\right|^{p} d x\right)^{1 / p} \\
& \quad+\frac{1}{h^{\beta}}\left(\frac{1}{w\left(x_{0}-h, x_{0}\right)} \int_{x_{0}}^{x_{0}+h}\left|M^{+} f_{2}(x)\right|^{p} d x\right)^{1 / p} \\
&=: \widetilde{I}+\widetilde{I I} .
\end{aligned}
$$

Using Theorem 1 and Lemma 11, the following is true:

$$
\begin{aligned}
\widetilde{I} & =\frac{1}{h^{\beta}}\left(\frac{1}{w\left(x_{0}-h, x_{0}\right)} \int_{x_{0}}^{x_{0}+h}\left|M^{+} f_{1}(x)\right|^{p} d x\right)^{1 / p} \\
& \leq \frac{1}{h^{\beta}}\left(\frac{1}{w\left(x_{0}-h, x_{0}\right)} \int_{x_{0}}^{x_{0}+2 h}|f(y)|^{p} d y\right)^{1 / p} \\
& \leq\left(\frac{w\left(x_{0}-2 h, x_{0}\right)}{w\left(x_{0}-h, x_{0}\right)}\right)^{1 / p}\|f\|_{\mathscr{M}_{p, \beta}^{+}(w)} \\
& \leq C\|f\|_{\mathscr{M}_{p, \beta}^{+}(w)} .
\end{aligned}
$$

Hölder's inequality and Proposition 12(a) allow us to estimate $\widetilde{I I}$ as

$$
\begin{aligned}
& \frac{1}{h^{\beta}}\left(\frac{1}{w\left(x_{0}-h, x_{0}\right)} \int_{x_{0}}^{x_{0}+h}\left|M^{+} f_{2}(x)\right|^{p} d x\right)^{1 / p} \\
& \leq \frac{1}{h^{\beta}} \frac{1}{w\left(x_{0}-h, x_{0}\right)^{1 / p}} \\
& \quad \times \sum_{j=1}^{\infty}\left(\frac{1}{2^{j}} \int_{x_{0}-2 h}^{x_{0}+2^{j+1} h}|f(y)|^{p} d y\right)^{1 / p} \\
& \leq \sum_{j=1}^{\infty} \frac{1}{2^{j(1 / p-\beta-1)}}\|f\|_{\mathscr{U}_{p, \beta}^{+}(w)} \\
& \leq C\|f\|_{\mathscr{M}_{p, \beta}^{+}(w)} .
\end{aligned}
$$

Theorem 7(b) can now be proven. Decomposing $f=f_{1}+$ $f_{2}=f \chi_{2 I}+f \chi_{(2 I)^{c}}$ shows that

$$
\begin{gathered}
\frac{1}{h^{\beta}}\left(\frac{1}{w\left(x_{0}-h, x_{0}\right)} \int_{x_{0}}^{x_{0}+h}\left|T^{+} f(y)-\left(T^{+} f\right)_{\left(x_{0}, x_{0}+h\right)}\right|^{p} d y\right)^{1 / p} \\
\leq \frac{2}{h^{\beta}}\left(\frac{1}{w\left(x_{0}-h, x_{0}\right)}\right. \\
\left.\quad \times \int_{x_{0}}^{x_{0}+h}\left|T^{+} f(y)-T^{+} f_{2}\left(x_{0}+2 h\right)\right|^{p} d y\right)^{1 / p} \\
\leq \frac{2}{h^{\beta}}\left(\frac{1}{w\left(x_{0}-h, x_{0}\right)} \int_{x_{0}}^{x_{0}+h}\left|T^{+} f_{1}(y)\right|^{p} d y\right)^{1 / p} \\
\quad+\frac{2}{h^{\beta}}\left(\frac{1}{w\left(x_{0}-h, x_{0}\right)}\right. \\
=: \bar{I}+\overline{I I} .
\end{gathered}
$$

The fact that, if $w \in A_{p}^{+}$, then $T^{+}$is bounded on $L^{p}(w)$ allows the following to be shown:

$$
\begin{aligned}
\bar{I} & \leq \frac{C}{h^{\beta}}\left(\frac{1}{w\left(x_{0}-h, x_{0}\right)} \int_{x_{0}}^{x_{0}+2 h}|f(y)|^{p} d y\right)^{1 / p} \\
& \leq C\left(\frac{w\left(x_{0}-2 h, x_{0}\right)}{w\left(x_{0}-h, x_{0}\right)}\right)^{1 / p}\|f\|_{\mathscr{M}_{p, \beta}^{+}(w)} \\
& \leq C\|f\|_{\mathscr{M}_{p, \beta}^{+}(w)}
\end{aligned}
$$

Here, Lemma 11 is used in the last inequality.

For the term $\overline{I I}$, by (11) and Proposition 12(a), we can derive the following:

$$
\begin{aligned}
\overline{I I} \leq & \frac{C}{h^{\beta} w\left(x_{0}-h, x_{0}\right)^{1 / p}} \\
& \times\left(\int_{x_{0}}^{x_{0}+h} \mid \int_{x_{0}+2 h}^{\infty}(K(y-z)\right. \\
\leq & \frac{\left.\left.C\left(x_{0}+2 h-z\right)\right)\left.f(z) d z\right|^{p} d y\right)^{1 / p}}{h^{\beta} w\left(x_{0}-h, x_{0}\right)^{1 / p}} \\
& \times\left(\int_{x_{0}}^{x_{0}+h}\left(\int_{x_{0}+2 h}^{\infty}\left|\frac{x_{0}+2 h-y}{\left(z-\left(x_{0}+h\right)\right)^{2}} f(z)\right| d z\right)^{p} d y\right)^{1 / p}
\end{aligned}
$$




$$
\begin{aligned}
\leq & C \frac{h^{1-\beta}}{w\left(x_{0}-h, x_{0}\right)^{1 / p}} \\
& \times\left(\int_{x_{0}}^{x_{0}+h}\left(\sum_{j=1}^{\infty} \int_{x_{0}+2^{j} h}^{x_{0}+2^{j+1} h}\left|\frac{f(z)}{\left(z-\left(x_{0}+h\right)\right)^{2}}\right| d z\right)^{p} d y\right)^{1 / p} \\
\leq & C \frac{h^{1+1 / p-\beta}}{w\left(x_{0}-h, x_{0}\right)^{1 / p}} \\
& \times \sum_{j=1}^{\infty} \frac{1}{\left(h\left(2^{j}-1\right)\right)^{2}} \int_{x_{0}+2^{j} h}^{x_{0}+2^{j+1} h}|f(z)| d z \\
\leq & C \frac{h^{1 / p-1-\beta}}{w\left(x_{0}-h, x_{0}\right)^{1 / p}} \\
& \times \sum_{j=1}^{\infty} \frac{1}{\left(2^{j}-1\right)^{2}}\left(\int_{x_{0}-h}^{x_{0}+2^{j+1} h}|f(z)|^{p} d z\right)^{1 / p}\left(2^{j} h\right)^{1 / p^{\prime}} \\
\leq & C\|f\|_{\mathscr{M}_{p, \beta}^{+}(w)} \sum_{j=1}^{\infty} \frac{1}{2^{j(1 / p-\beta)}} \\
\leq & C\|f\|_{\mathscr{M}_{p, \beta}^{+}(w) \cdot}
\end{aligned}
$$

On account of the estimates for $\bar{I}$ and $\overline{I I}$ given above, the following can be proved:

$$
\left\|T^{+} f\right\|_{\mathscr{C}_{p, \beta}^{+}(w)} \leq C\|f\|_{\mathscr{U}_{p, \beta}^{+}(w)} .
$$

Proof of Theorem 8. We begin with the proof for $M_{\alpha}^{+}$, which is similar to that of Theorem 7(a). It is sufficient to show that there exists constant $C>0$ such that

$$
\begin{aligned}
& \frac{1}{h^{\beta}}\left(\frac{1}{w\left(x_{0}-h, x_{0}\right)^{q}} \int_{x_{0}}^{x_{0}+h}\left|M_{\alpha}^{+} f(y)\right|^{q} d y\right)^{1 / q} \\
& \quad \leq C\|f\|_{\mathscr{U}_{p, \beta}^{+}\left(w^{p}\right)}
\end{aligned}
$$

Decompose $f=f_{1}+f_{2}=f \chi_{2 I}+f \chi_{(2 I)^{c}}$ to obtain

$$
\begin{aligned}
\frac{1}{h^{\beta}}( & \left.\frac{1}{w\left(x_{0}-h, x_{0}\right)^{q}} \int_{x_{0}}^{x_{0}+h}\left|M_{\alpha}^{+} f(x)\right|^{q} d x\right)^{1 / q} \\
\leq & \frac{1}{h^{\beta}}\left(\frac{1}{w\left(x_{0}-h, x_{0}\right)^{q}} \int_{x_{0}}^{x_{0}+h}\left|M_{\alpha}^{+} f_{1}(x)\right|^{q} d x\right)^{1 / q} \\
& +\frac{1}{h^{\beta}}\left(\frac{1}{w\left(x_{0}-h, x_{0}\right)^{q}} \int_{x_{0}}^{x_{0}+h}\left|M_{\alpha}^{+} f_{2}(x)\right|^{q} d x\right)^{1 / q} \\
= & : \widetilde{J}+\widetilde{J J} .
\end{aligned}
$$

By Theorem 4 and Lemma 11,

$$
\begin{aligned}
\widetilde{J} & =\frac{1}{h^{\beta}}\left(\frac{1}{w\left(x_{0}-h, x_{0}\right)^{q}} \int_{x_{0}}^{x_{0}+h}\left|M_{\alpha}^{+} f(x)\right|^{q} d x\right)^{1 / q} \\
& \leq C\|f\|_{\mathcal{U}_{p, \beta}^{+}\left(w^{p}\right)} .
\end{aligned}
$$

By the same arguments as those of $\widetilde{I I}, \widetilde{J J}$ can be estimated as

$$
\begin{aligned}
& \frac{1}{h^{\beta}}\left(\frac{1}{w\left(x_{0}-h, x_{0}\right)^{q}} \int_{x_{0}}^{x_{0}+h}\left|M_{\alpha}^{+} f_{2}(x)\right|^{q} d x\right)^{1 / q} \\
& \quad \leq \sum_{j=1}^{\infty} \frac{1}{2^{j(1 / q-\beta-1)}}\|f\|_{\mathscr{M}_{p, \beta}^{+}(w)} \\
& \quad \leq C\|f\|_{\mathscr{M}_{p, \beta}^{+}\left(w^{p}\right)} .
\end{aligned}
$$

The proof of (b) is a reprise of the argument given in the proof of Theorem 7(b). Set $f=f_{1}+f_{2}=f \chi_{2 I}+f \chi_{(2 I)^{c}}$ to obtain

$$
\begin{aligned}
& \frac{1}{h^{\beta}}\left(\frac{1}{w\left(x_{0}-h, x_{0}\right)^{q}}\right. \\
& \left.\quad \times \int_{x_{0}}^{x_{0}+h}\left|I_{\alpha}^{+} f(y)-\left(I_{\alpha}^{+} f\right)_{\left(x_{0}, x_{0}+h\right)}\right|^{q} d y\right)^{1 / q} \\
& \leq \frac{2}{h^{\beta}}\left(\frac{1}{w\left(x_{0}-h, x_{0}\right)^{q}}\right. \\
& \left.\leq \frac{2}{h^{\beta}}\left(\frac{1}{w\left(x_{0}-h, x_{0}\right)^{q}} \int_{x_{0}}^{x_{0}+h}\left|I_{\alpha}^{+} f_{1}(y)\right|^{q} d y\right)^{1 / q}\left|I_{\alpha}^{+} f(y)-I_{\alpha}^{+} f_{2}\left(x_{0}+2 h\right)\right|^{q} d y\right)^{1 / q} \\
& \quad+\frac{2}{h^{\beta}}\left(\frac{1}{w\left(x_{0}-h, x_{0}\right)^{q}}\right. \\
& \left.=: J+J J . \quad \times \int_{x_{0}}^{x_{0}+h}\left|I_{\alpha}^{+} f_{2}(y)-I_{\alpha}^{+} f_{2}\left(x_{0}+2 h\right)\right|^{q} d y\right)^{1 / q}
\end{aligned}
$$

Theorem 4 and Lemma 11 allow us to estimate $J$ as

$$
\begin{aligned}
J & \leq \frac{C}{h^{\beta}} \frac{1}{w\left(x_{0}-h, x_{0}\right)}\left(\int_{x_{0}}^{x_{0}+2 h}|f(y)|^{p} d y\right)^{1 / p} \\
& \leq C\left(\frac{w\left(x_{0}-2 h, x_{0}\right)}{w\left(x_{0}-h, x_{0}\right)}\right)\|f\|_{\mathscr{M}_{p, \beta}^{+}(w)} \\
& \leq C\|f\|_{\mathscr{M}_{p, \beta}^{+}\left(w^{p}\right)} .
\end{aligned}
$$


In view of

$$
\begin{aligned}
& \left|I_{\alpha}^{+} f_{2}(y)-I_{\alpha}^{+} f_{2}\left(x_{0}+2 h\right)\right| \\
& \leq \int_{x_{0}+2 h}^{\infty}\left|\frac{1}{|z-y|^{1-\alpha}}-\frac{1}{\left|z-\left(x_{0}+2 h\right)\right|^{1-\alpha}}\right| \\
& \quad \times|f(z)| d z \\
& \leq C \int_{x_{0}+2 h}^{\infty} \frac{\left|x_{0}+2 h-y\right|}{\left|z-\left(x_{0}+2 h\right)\right|^{2-\alpha}}|f(z)| d z,
\end{aligned}
$$

we obtain by Hölder's inequality and Proposition 12(b) that

$$
\begin{aligned}
J J \leq & C \frac{h^{1+1 / q-\beta}}{w\left(x_{0}-h, x_{0}\right)} \sum_{j=1}^{\infty} \int_{x_{0}+2^{j} h}^{x_{0}+2^{j+1} h} \frac{|f(z)|}{\left(z-\left(x_{0}+h\right)\right)^{2-\alpha}} d z \\
\leq & C \frac{h^{1+1 / q-\beta}}{w\left(x_{0}-h, x_{0}\right)} \sum_{j=1}^{\infty} \frac{1}{\left(h\left(2^{j}-1\right)\right)^{2-\alpha}} \\
& \times \int_{x_{0}-h}^{x_{0}+2^{j+1} h}|f(z)| d z \\
\leq & C \frac{h^{\alpha+1 / q-1-\beta}}{w\left(x_{0}-h, x_{0}\right)} \\
& \times \sum_{j=1}^{\infty} \frac{1}{\left(2^{j}-1\right)^{2}}\left(\int_{x_{0}-h}^{x_{0}+2^{j+1} h}|f(z)|^{p} d z\right)^{1 / p}\left(2^{j} h\right)^{1 / p^{\prime}} \\
\leq & C\|f\|_{\mathscr{M}_{p, \beta}^{+}\left(w^{p}\right)}^{\infty} \sum_{j=1}^{\infty} \frac{1}{2^{j(1 / p-\alpha-\beta)}} \\
\leq & C\|f\|_{\mathscr{M}_{p, \beta}^{+}\left(w^{p}\right)} \cdot
\end{aligned}
$$

We have thus proved Theorem 8 .

\section{Boundedness of One-Sided Sublinear Operators}

The method used in the proof of Theorem 7(b) depends heavily on the convolution of the kernel function. However, there are some other one-sided operators with nonconvoluted kernels. Such operators appear in many places and PDEs. The one-sided oscillatory singular integral operators that were first introduced by the authors of this paper in a previous study are one such example [7].

A class of more general one-sided operators that do not necessarily have convolution kernels can now be studied. Let $D_{k}=2^{k} I$ and $A_{k}=D_{k} \backslash D_{k-1}$ for $k \in Z$. In this section, a definition made in a previous study [30] can be adopted to introduce a one-sided sublinear operator satisfying the following size condition:

$$
\left|\mathscr{T}^{+} f(x)\right| \leq \frac{C}{2^{k} h}\|f\|_{L^{1}\left(A_{k}\right)},
$$

where supp $f \subseteq A_{k}$ and $0 \leq x_{0}<x \leq x_{0}+2^{k-1} h$ with $k \in Z$.
For the fractional case, the corresponding size condition can be introduced:

$$
\left|\mathscr{T}_{\alpha}^{+} f(x)\right| \leq \frac{C}{\left(2^{k} h\right)^{1-\alpha}}\|f\|_{L^{1}\left(A_{k}\right)}, \quad 0<\alpha<1,
$$

where supp $f \subseteq A_{k}$ and $0 \leq x_{0}<x \leq x_{0}+2^{k-1} h$ with $k \in Z$.

It is easy to confirm that the condition (45) is satisfied by $M^{+}, T^{+}$and the one-sided oscillatory singular integral operators and both $M_{\alpha}^{+}$and $I_{\alpha}^{+}$satisfy (46).

Theorem 13. Let $-1 / p \leq \beta<0,1<p<1 /(1+\beta), w \in A_{p}^{+}$, and the one-sided sublinear operator $\mathscr{T}^{+}$satisfy (45). Then if $\mathscr{T}^{+}$is bounded on $L^{p}(w), \mathscr{T}^{+}$is bounded on $\mathscr{M}_{p, \beta}^{+}(w)$.

Theorem 14. Let $0<\alpha<1,-1 / q \leq \beta<0,1 / q=1 / p-\alpha$, $p<$ $q<1 /(1+\beta), w \in A_{(p, q)}^{+}$, and the one-sided sublinear operator $\mathscr{T}_{\alpha}^{+}$satisfy (46). If $\mathscr{T}_{\alpha}^{+}$is bounded from $L^{p}\left(w^{p}\right)$ to $L^{q}\left(w^{q}\right)$, then $\mathscr{T}_{\alpha}^{+}$is bounded from $\mathscr{M}_{p, \beta}^{+}\left(w^{p}\right)$ to $\mathscr{M}_{q, \beta}^{+}\left(w^{q}\right)$.

Theorems 13 and 14 agree with Theorems 7(a) and 8(a) but are different from Theorems 7(b) and 8(b). The conditions of the kernel functions in Theorems 13 and 14 are weaker than those of Theorems 7(b) and 8(b), respectively, in that only the size conditions are used there. For this reason, Theorems 13 and 14 can be seen as an extension of Theorems 7 and 8 , respectively. However, the present study was conducted under the assumptions that $1<p<1 /(1+\beta)$ and $p<$ $q<1 /(1+\beta)$. These conditions are stronger than those of Theorems 7 (b) and 8(b).

Proof of Theorem 13. It is sufficient to show that there exists $C>0$ such that

$$
\frac{1}{h^{\beta}}\left(\frac{1}{w\left(x_{0}-h, x_{0}\right)} \int_{x_{0}}^{x_{0}+h}\left|\mathscr{T}^{+} f(x)\right|^{p} d x\right)^{1 / p} \leq C\|f\|_{\mathscr{M}_{p, \beta}^{+}(w)}
$$

$f=f_{1}+f_{2}=f \chi_{2 I}+f \chi_{(2 I)^{c}}$ is decomposed to produce

$$
\begin{aligned}
& \frac{1}{h^{\beta}}\left(\frac{1}{w\left(x_{0}-h, x_{0}\right)} \int_{x_{0}}^{x_{0}+h}\left|\mathscr{T}^{+} f(x)\right|^{p} d x\right)^{1 / p} \\
& \leq \frac{1}{h^{\beta}}\left(\frac{1}{w\left(x_{0}-h, x_{0}\right)} \int_{x_{0}}^{x_{0}+h}\left|\mathscr{T}^{+} f_{1}(x)\right|^{p} d x\right)^{1 / p} \\
& \quad+\frac{1}{h^{\beta}}\left(\frac{1}{w\left(x_{0}-h, x_{0}\right)} \int_{x_{0}}^{x_{0}+h}\left|\mathscr{T}^{+} f_{2}(x)\right|^{p} d x\right)^{1 / p} \\
& =: K+K K .
\end{aligned}
$$


Using the fact that $\mathscr{T}^{+}$is bounded on $L^{p}(w)$,

$$
\begin{aligned}
K & \leq \frac{C}{h^{\beta}}\left(\frac{1}{w\left(x_{0}-h, x_{0}\right)} \int_{x_{0}}^{x_{0}+2 h}|f(y)|^{p} d y\right)^{1 / p} \\
& \leq C\left(\frac{w\left(x_{0}-2 h, x_{0}\right)}{w\left(x_{0}-h, x_{0}\right)}\right)^{1 / p}\|f\|_{\mathscr{M}_{p, \beta}^{+}(w)} \\
& \leq C\|f\|_{\mathscr{M}_{p, \beta}^{+}(w)}
\end{aligned}
$$

can be found easily.

In view of (45), the following is true:

$$
\begin{aligned}
\left|\mathscr{T}^{+} f_{2}(x)\right| \leq C \sum_{k=1}^{\infty} \frac{1}{2^{k} h} \int_{x_{0}+2^{k} h}^{x_{0}+2^{k+1} h}|f(y)| d y \\
\leq C \sum_{k=1}^{\infty} \frac{1}{2^{k} h}\left(\int_{x_{0}-h}^{x_{0}+2^{k+1} h}|f(y)|^{p} d y\right)^{1 / p} \\
\times\left(2^{k} h\right)^{1 / p^{\prime}} \\
\leq C \sum_{k=1}^{\infty} \frac{w\left(x_{0}-2^{k+1} h, x_{0}\right)^{1 / p}}{\left(2^{k} h\right)^{1 / p-\beta}}\|f\|_{\mathscr{M}_{p, \beta}^{+}(w)}
\end{aligned}
$$

Using Proposition 12, KK can be estimated as

$$
\begin{aligned}
K K \leq & C\|f\|_{\mathscr{M}_{p, \beta}^{+}(w)} \\
& \times \sum_{k=1}^{\infty} \frac{h^{1 / p-\beta}}{\left(2^{k} h\right)^{1 / p-\beta}}\left(\frac{w\left(x_{0}-2^{k+1} h, x_{0}-h\right)}{w\left(x_{0}-h, x_{0}\right)}\right)^{1 / p} \\
\leq & C\|f\|_{\mathscr{M}_{p, \beta}^{+}(w)} \sum_{k=1}^{\infty} \frac{1}{2^{k(1 / p-\beta-1)}} \\
\leq & C\|f\|_{\mathscr{M}_{p, \beta}^{+}(w)} .
\end{aligned}
$$

Proof of Theorem 14. An argument similar to that used in the proof of Theorem 13 can be used to produce

$$
\begin{gathered}
\frac{1}{h^{\beta}}\left(\frac{1}{w\left(x_{0}-h, x_{0}\right)^{q}} \int_{x_{0}}^{x_{0}+h}\left|\mathscr{T}_{\alpha}^{+} f(x)\right|^{q} d x\right)^{1 / q} \\
\leq \frac{1}{h^{\beta}}\left(\frac{1}{w\left(x_{0}-h, x_{0}\right)^{q}}\right. \\
\left.\quad \times \int_{x_{0}}^{x_{0}+h}\left|\mathscr{T}_{\alpha}^{+} f_{1}(x)\right|^{q} d x\right)^{1 / q} \\
+\frac{1}{h^{\beta}}\left(\frac{1}{w\left(x_{0}-h, x_{0}\right)^{q}}\right. \\
\left.\quad \times \int_{x_{0}}^{x_{0}+h}\left|\mathscr{T}_{\alpha}^{+} f_{2}(x)\right|^{q} d x\right)^{1 / q} \\
=: L+L L .
\end{gathered}
$$

Estimating the term $L$, using Lemma 11 and the fact that $\mathscr{T}_{\alpha}^{+}$ is bounded from $L^{p}\left(w^{p}\right)$ to $L^{q}\left(w^{q}\right)$ produce

$$
\begin{aligned}
L & \leq \frac{C}{h^{\beta}}\left(\frac{1}{w\left(x_{0}-h, x_{0}\right)^{p}} \int_{x_{0}}^{x_{0}+2 h}|f(y)|^{p} d y\right)^{1 / p} \\
& \leq C\left(\frac{w\left(x_{0}-2 h, x_{0}\right)}{w\left(x_{0}-h, x_{0}\right)}\right)\|f\|_{\mathscr{M}_{p, \beta}^{+}\left(w^{p}\right)} \\
& \leq C\|f\|_{\mathscr{M}_{p, \beta}^{+}\left(w^{p}\right)^{.}}
\end{aligned}
$$

The term $L L$ (46) facilitates the production of

$$
\begin{aligned}
\left|\mathscr{T}_{\alpha}^{+} f_{2}(x)\right| \leq & C \sum_{k=1}^{\infty} \frac{1}{\left(2^{k} h\right)^{1-\alpha}} \int_{x_{0}+2^{k} h}^{x_{0}+2^{k+1} h}|f(y)| d y \\
\leq & C \sum_{k=1}^{\infty} \frac{1}{\left(2^{k} h\right)^{1-\alpha}} \\
& \times\left(\int_{x_{0}-h}^{x_{0}+2^{k+1} h}|f(y)|^{p} d y\right)^{1 / p} \\
& \times\left(2^{k} h\right)^{1 / p^{\prime}} \\
\leq & C \sum_{k=1}^{\infty} \frac{w\left(x_{0}-2^{k+1} h, x_{0}-h\right)}{\left(2^{k} h\right)^{1 / p-\beta-\alpha}} \\
& \times\|f\|_{\mathscr{M}_{p, \beta}^{+}\left(w^{p}\right)} .
\end{aligned}
$$

Therefore,

$$
\begin{aligned}
L L \leq & C\|f\|_{\mathscr{M}_{p, \beta}^{+}\left(w^{p}\right)} \\
& \times \sum_{k=1}^{\infty} \frac{h^{1 / q-\beta}}{\left(2^{k} h\right)^{1 / q-\beta}}\left(\frac{w\left(x_{0}-2^{k+1} h, x_{0}-h\right)}{w\left(x_{0}-h, x_{0}\right)}\right) \\
\leq & C\|f\|_{\mathscr{M}_{p, \beta}^{+}\left(w^{p}\right)} \sum_{k=1}^{\infty} \frac{1}{2^{k(1 / q-\beta-1)}} \\
\leq & C\|f\|_{\mathscr{M}_{p, \beta}^{+}\left(w^{p}\right)}
\end{aligned}
$$

where we have used Propositions 10 and 12.

\section{Conflict of Interests}

The authors declare that there is no conflict of interests regarding the publication of this paper.

\section{Acknowledgments}

The authors cordially thank the referees for their careful reading and helpful comments. This work was partially supported by NSF of China (Grants nos. 11301249, 11271175, and 1171345), NSF of Shandong Province (Grant no. ZR2012AQ026), and AMEP of Linyi University. 


\section{References}

[1] F. J. Martín-Reyes, P. Ortega Salvador, and A. de la Torre, "Weights for one-sided operators," in Recent Development in Real and Harmonic Analysis, Applied and Numerical Harmonic Analysis, Birkhäuser, Boston, Mass, USA, 2009.

[2] P. Butzer and U. Westphal, "Introduction to fractional calculus," in Fractional Calculus, Applications in Physics, H. Hilfer, Ed., pp. 1-85, World Scientific, Singapore, 2000.

[3] Z. W. Fu, S. Z. Lu, and W. Yuan, "A weighted variant of Riemann-Liouville fractional integrals on $\mathbb{R}^{n}$, , Abstract and Applied Analysis, vol. 2012, Article ID 780132, 18 pages, 2012.

[4] E. Sawyer, "Weighted inequalities for the one-sided HardyLittlewood maximal functions," Transactions of the American Mathematical Society, vol. 297, no. 1, pp. 53-61, 1986.

[5] H. Aimar, L. Forzani, and F. J. Martín-Reyes, "On weighted inequalities for singular integrals," Proceedings of the American Mathematical Society, vol. 125, no. 7, pp. 2057-2064, 1997.

[6] K. F. Andersen and E. T. Sawyer, "Weighted norm inequalities for the Riemann-Liouville and Weyl fractional integral operators," Transactions of the American Mathematical Society, vol. 308, no. 2, pp. 547-558, 1988.

[7] Z. Fu, S. Lu, S. Sato, and S. Shi, "On weighted weak type norm inequalities for one-sided oscillatory singular integrals," Studia Mathematica, vol. 207, no. 2, pp. 137-151, 2011.

[8] F. J. Martín-Reyes, P. Ortega Salvador, and A. de la Torre, "Weighted inequalities for one-sided maximal functions," Transactions of the American Mathematical Society, vol. 319, no. 2, pp. 517-534, 1990.

[9] F. J. Martín-Reyes and A. de la Torre, "One-sided BMO spaces," Journal of the London Mathematical Society, vol. 49, no. 3, pp. 529-542, 1994.

[10] F. J. Martín-Reyes and A. de la Torre, "Two weight norm inequalities for fractional one-sided maximal operators," Proceedings of the American Mathematical Society, vol. 117, no. 2, pp. 483-489, 1993.

[11] H. Aimar and R. Crescimbeni, "On one-sided BMO and Lipschitz functions," Annali della Scuola Normale Superiore di Pisa Serie IV, vol. 27, no. 3-4, pp. 437-456, 1998.

[12] Z. W. Fu and S. Z. Lu, "One-sided Triebel-Lizorkin space and its applications," Scientia Sinica Mathematica, vol. 41, pp. 43-52, 2011.

[13] S. Ombrosi and L. de Rosa, "Boundedness of the Weyl fractional integral on one-sided weighted Lebesgue and Lipschitz spaces," Publicacions Matemàtiques, vol. 47, no. 1, pp. 71-102, 2003.

[14] L. de Rosa and C. Segovia, "Equivalence of norms in one-sided $H^{p}$ spaces," Collectanea Mathematica, vol. 53, no. 1, pp. 1-20, 2002.

[15] P. G. Lemarié-Rieusset, "The Navier-Stokes equations in the critical Morrey-Campanato space," Revista Matemática Iberoamericana, vol. 23, no. 3, pp. 897-930, 2007.

[16] G. Lu, "Embedding theorems on Campanato-Morrey spaces for vector fields and applications," Comptes Rendus de l'Académie des Sciences. Série I., vol. 320, no. 4, pp. 429-434, 1995.

[17] G. Lu, "Embedding theorems on Campanato-Morrey spaces for vector fields on Hörmander type," Approximation Theory and Its Applications, vol. 14, no. 1, pp. 69-80, 1998.

[18] H. Triebel, Theory of Function Spaces. II, vol. 84 of Monographs in Mathematics, Birkhäuser, Basel, Switzerland, 1992.

[19] X. T. Duong, J. Xiao, and L. Yan, "Old and new Morrey spaces with heat kernel bounds," The Journal of Fourier Analysis and Applications, vol. 13, no. 1, pp. 87-111, 2007.
[20] H. Lin, E. Nakai, and D. Yang, "Boundedness of Lusin-area and $g_{\lambda}^{*}$ functions on localized Morrey-Campanato spaces over doubling metric measure spaces," Journal of Function Spaces and Applications, vol. 9, no. 3, pp. 245-282, 2011.

[21] D. Yang, D. Yang, and Y. Zhou, "Localized Morrey-Campanato spaces on metric measure spaces and applications to Schrödinger operators," Nagoya Mathematical Journal, vol. 198, pp. 77-119, 2010.

[22] C. B. Morrey Jr., "On the solutions of quasi-linear elliptic partial differential equations," Transactions of the American Mathematical Society, vol. 43, no. 1, pp. 126-166, 1938.

[23] D. Fan, Y. Pan, and D. Yang, "A weighted norm inequality for rough singular integrals," The Tohoku Mathematical Journal, vol. 51, no. 2, pp. 141-161, 1999.

[24] D. Fan, S. Lu, and D. Yang, "Boundedness of operators in Morrey spaces on homogeneous spaces and its applications," Acta Mathematica Sinica, vol. 14, pp. 625-634, 1998.

[25] W. Yuan, W. Sickel, and D. Yang, Morrey and Campanato meet Besov, Lizorkin and Triebel, vol. 2005 of Lecture Notes in Mathematics, Springer, Berlin, Germany, 2010.

[26] R. R. Coifman and C. Fefferman, "Weighted norm inequalities for maximal functions and singular integrals," Polska Akademia Nauk, vol. 51, pp. 241-250, 1974.

[27] P. Guan and E. Sawyer, "Regularity estimates for the oblique derivative problem," Annals of Mathematics, vol. 137, no. 1, pp. 1-70, 1993.

[28] L. De Rosa and C. Segovia, "Dual spaces for one-sided weighted Hardy spaces," Revista de la Unión Matemática Argentina, vol. 40, no. 3-4, pp. 49-71, 1997.

[29] M. S. Riveros and A. de la Torre, "On the best ranges for $A_{p}^{+}$and $\mathrm{RH}_{r}^{+}$," Czechoslovak Mathematical Journal, vol. 51, no. 2, pp. 285-301, 2001.

[30] X. W. Li and D. C. Yang, "Boundedness of some sublinear operators on Herz spaces," Illinois Journal of Mathematics, vol. 40, no. 3, pp. 484-501, 1996. 


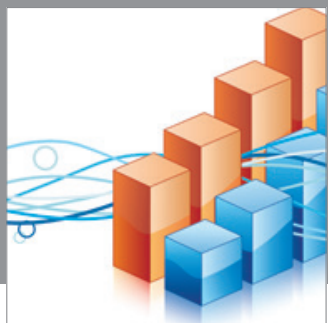

Advances in

Operations Research

mansans

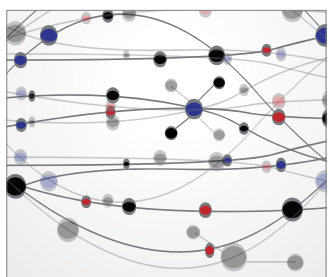

The Scientific World Journal
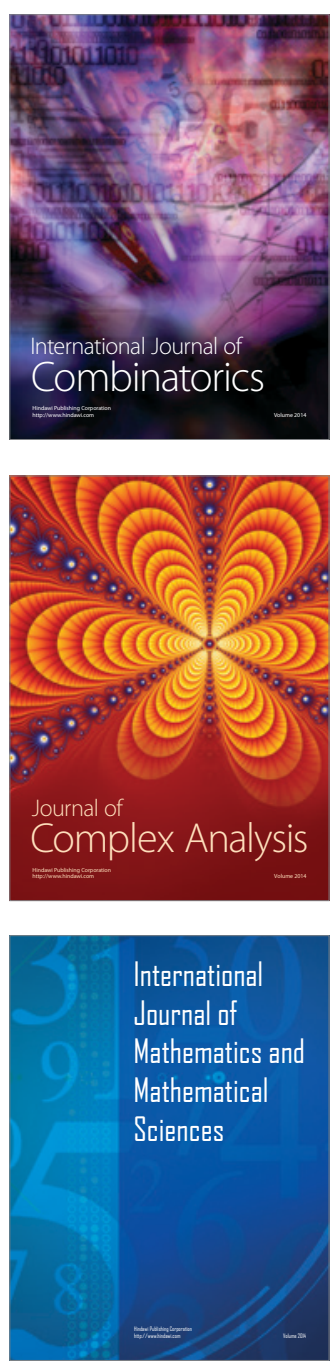
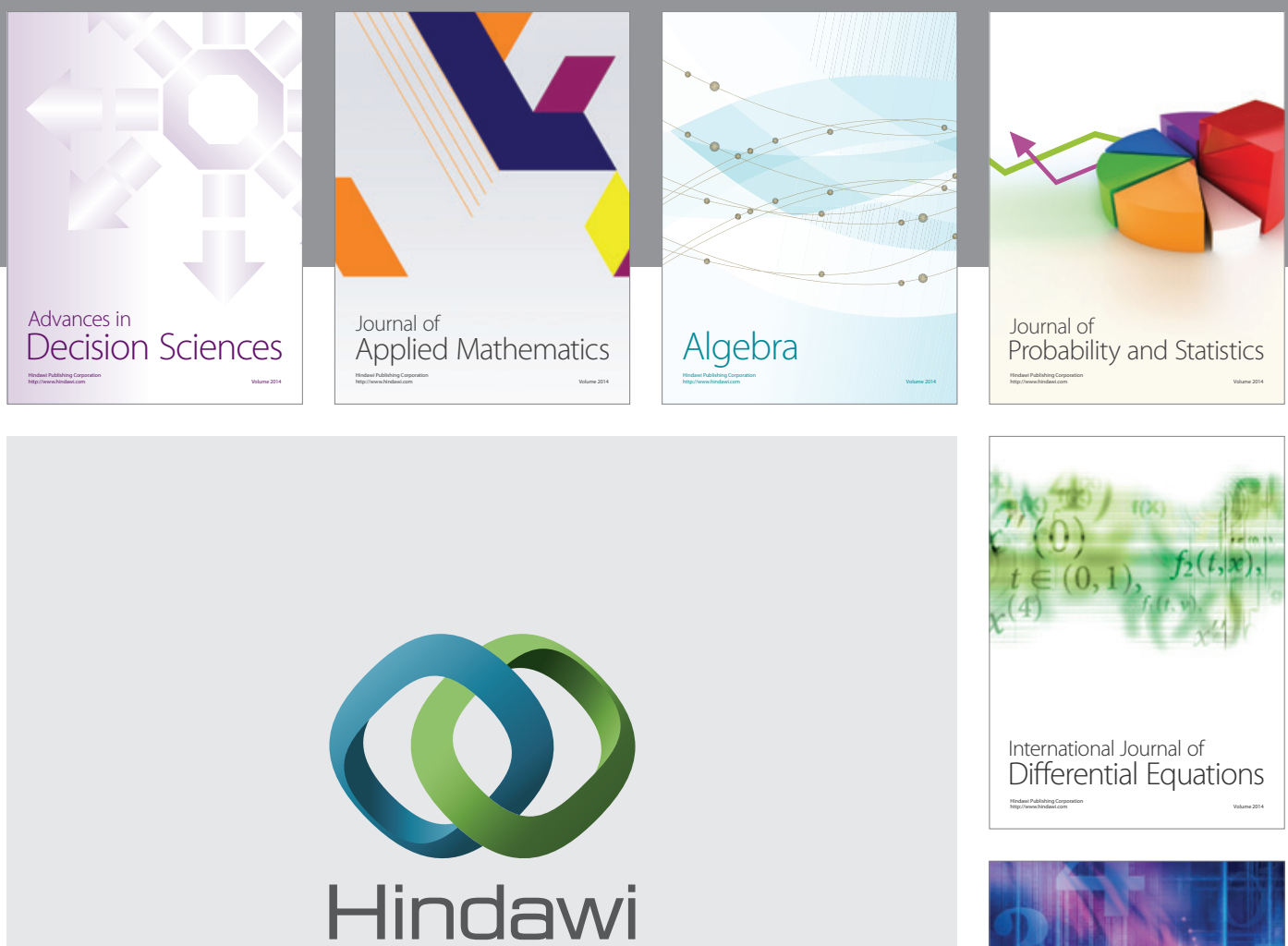

Submit your manuscripts at http://www.hindawi.com
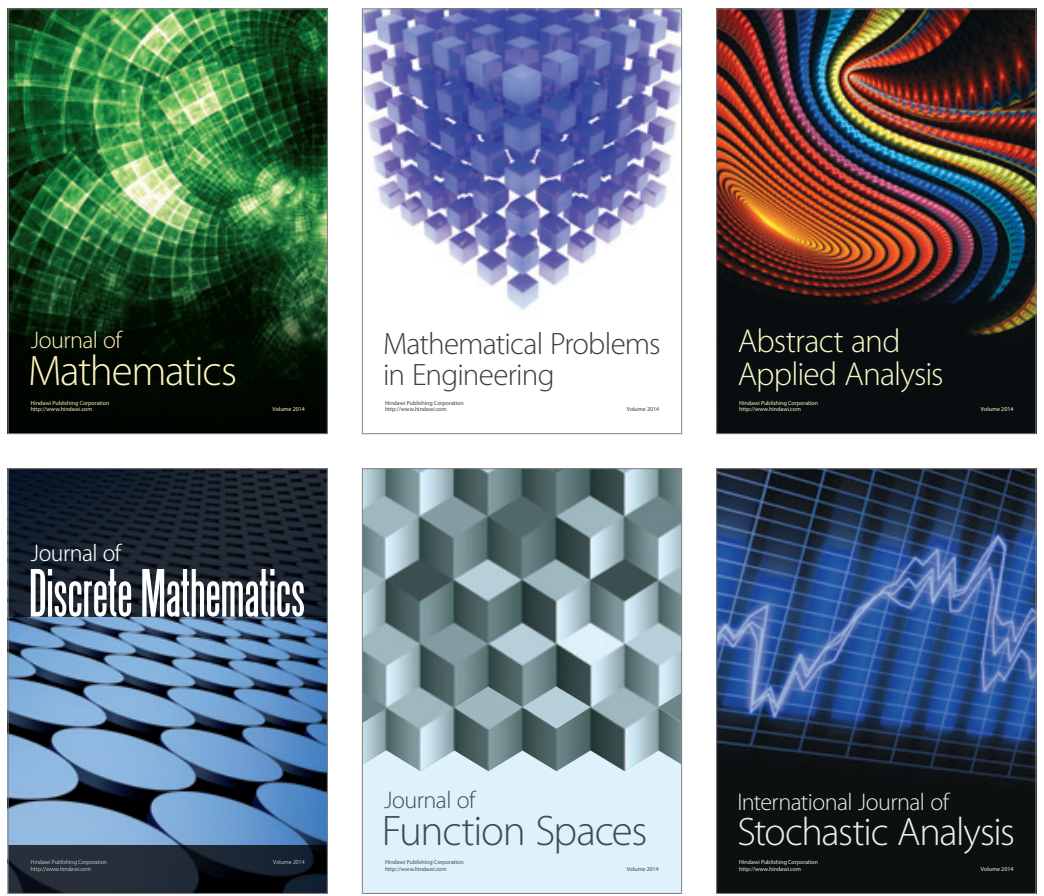

Journal of

Function Spaces

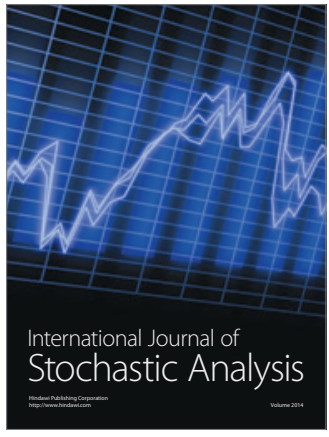

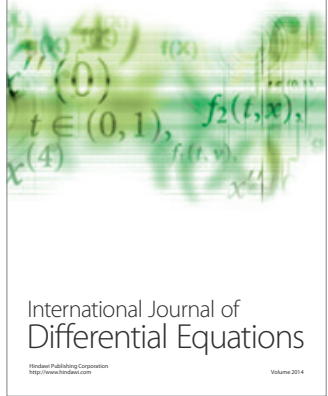
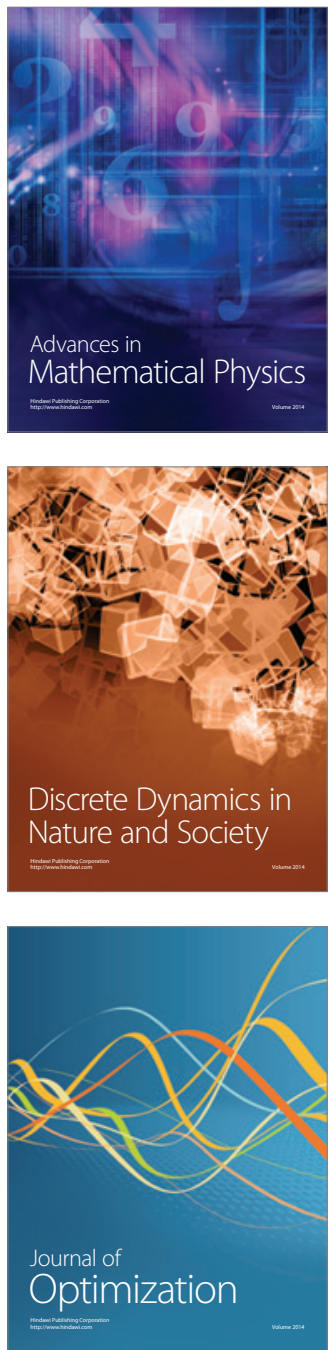